\section{Molecular Syndromology}

Mol Syndromol 2018;9:279-286

DOI: $10.1159 / 000494231$
Accepted: August 27, 2018

by M. Schmid

Published online: October 30, 2018

\title{
Paving the Way for Therapy: The Second International Conference of the Trisomy 21 Research Society
}

\author{
Roger H. Reeves ${ }^{\text {a }}$ Jean Delabar ${ }^{f}$ Marie-Claude Potier ${ }^{f}$ \\ Anita Bhattacharyya $^{\text {b }}$ Elizabeth Head $^{c}$ Cynthia Lemere $^{d}$ Alain D. Dekker ${ }^{g}$ \\ Peter De Deyn $^{g}$ Pablo Caviedes $^{\text {h }}$ Mara Dierssen $^{\text {i }}$ Jorge Busciglio $^{\text {e }}$ \\ a Johns Hopkins University School of Medicine, Baltimore, MD, bWaisman Center, University of Wisconsin-Madison, \\ Madison, WI, 'Sanders Brown Center on Aging, University of Kentucky, Lexington, KY, 'Harvard Medical School, \\ Boston, MA, and ${ }^{\mathrm{e}}$ University of California-Irvine, Irvine, CA, USA; ${ }^{\mathrm{f}}$ Brain and Spine Institute, Paris, France; ${ }^{9}$ University \\ of Groningen and University Medical Center, Groningen, The Netherlands; ${ }^{\mathrm{h}}$ Faculty of Medicine, University of Chile, \\ Santiago, Chile; 'Center for Genomic Regulation, Barcelona, Spain
}

\section{Keywords}

Alzheimer disease · Down syndrome · Trisomy 21 Research Society

\begin{abstract}
In the last decade, a number of important research advances in different fields have allowed Down syndrome (DS) research to flourish, creating a time of both unparalleled opportunity and considerable challenge. Building a scientific framework that distills mechanisms involved in the developmental intellectual disability of DS as well as the early-onset component of Alzheimer disease and the several other comorbidities associated with the condition is a challenge that scientists are now tackling using novel technologies and multidisciplinary approaches. The Trisomy 21 Research Society (T21RS) was founded in 2014 to address these evolving needs and challenges. In June of 2017, the T21RS held its 2nd International Conference in Chicago, USA. With more than 200 scientists, advocates, people with DS, and family members in attendance, the meeting served as a forum for the
\end{abstract}

\section{KARGER}

(C) 2018 S. Karger AG, Basel

E-Mail karger@karger.com

www.karger.com/msy discussion of the latest research and clinical advances as well as the most compelling needs of people with DS and their families.

(c) 2018 S. Karger AG, Basel

Down syndrome (DS, trisomy 21) is characterized by specific structural changes in the brain, a range of cognitive deficits and intellectual disability, a very high susceptibility to develop Alzheimer disease (AD) by middle age, and several clinical conditions including a high frequency of childhood leukemia, a low frequency of adult cancers, reduced atherosclerotic disease, craniofacial dysmorphology, congenital heart disease, and immune and endocrine abnormalities. Although DS is the most common genetic cause of intellectual impairment, surprisingly little is known about the specific defects in neuronal development and function that cause intellectual disability. Similarly, the underlying mechanisms that trigger premature aging and Alzheimer dementia in DS individuals are currently unclear. This lack of knowledge hinders our

Jorge Busciglio

University of California-Irvine

BioSci III, Room 3216

Irvine, CA 92697 (USA)

E-Mail jbuscigl@uci.edu 
ability to translate research findings to therapies for individuals with DS and demonstrates the need for further research in DS.

The Trisomy 21 Research Society (T21RS) is the first international nonprofit organization aimed at stimulating DS research in the broadest sense, including basic, translational, and clinical areas. The mission of the T21RS is to advance research in DS, and this requires establishing programs to support and strengthen the field by bringing together scientists of diverse backgrounds, illuminating the most pressing clinical issues, facilitating the integration of research directed at all levels of biological organization, and encouraging the application of new scientific knowledge to develop improved treatments for persons with DS, placing greater emphasis on meeting patient needs while optimizing for quality, safety, and regulatory compliance. The participation of individuals with DS and the support of families as well as advocate groups, with strong emphasis on exchange of information, are thus key to accelerate research with the common goal of developing effective therapies to improve general health and quality of life of persons with DS.

The 2nd International Conference of the T21RS was held at the Feinberg School of Medicine of Northwestern University in Chicago, from June 7 to 11 of 2017 with 223 registered participants from 19 different countries. Here, we summarize some of the most relevant scientific presentations, highlighting current basic and translational research as well as the "Science \& Society Symposium" where scientists, advocates, and families addressed some of the most compelling needs of the people affected by DS.

\section{Novel Research into Disease Mechanisms in Down Syndrome}

Tarik Haydar (USA) and Nenad Sestan (USA) gave 2 related presentations focused on emerging data linked to dysmyelination in DS during brain development. Dr. Haydar presented the cellular, molecular, and in vivo studies in the Ts65Dn mouse model that identified a cellautonomous defect in oligodendrocyte differentiation, demonstrating that fewer mature oligodendrocytes are produced in the forebrain white matter tracts. These cellular defects lead to reductions in myelin thickness and node of Ranvier frequency, both of which lead to slower action potential conduction velocity in the white matter tracts. Dr. Sestan highlighted novel genomic data acquired from a series of human postmortem brain samples from late prenatal gestation to adulthood that identify gene networks that are altered in the dorsolateral prefrontal cortex and the cerebellum, among other brain areas. The data demonstrated that key gene networks involved in oligodendrocyte differentiation are also altered in human brain development, providing an important and strong parallel between the mouse model and human studies.

Pablo Helguera (Argentina) described a stereotyped phenotype of oxidative stress and metabolic dysfunction among human autosomal trisomies including Down, Edward, and Patau syndromes. Interestingly, despite their obvious genotypic differences, primary cells from these aneuploidies activate common antioxidant responses. Treatments with mitochondria-targeted antioxidants rescued both cellular metabolism and function. The results indicated that in addition to decreasing oxidative damage, energy metabolism is a primary therapeutic target for aneuploidies in general and DS in particular.

Stephanie Sherman (USA) discussed the metabolomic analysis carried out on a number of amniotic fluid samples from second trimester pregnancies (39 trisomy 21 and 80 euploid controls). The results showed an enrichment in metabolites involved in steroid, lipid, nucleotide, and amino acid metabolism in trisomy 21 samples. Their role in the DS phenotype remains to be studied.

Silencing one chromosome 21 in DS was recently demonstrated in cellular models of trisomy 21 by inserting the gene encoding the $\mathrm{X}$-inactivated specific transcript (XIST) in one chromosome 21 [Jiang et al., 2013]. Building on these results, Jen-Chieh Chiang (USA) presented data showing that overproduction of megakaryocytes in cell cultures undergoing hematopoiesis leading to megakaryocytic leukemia in DS was corrected by XIST-mediated chromosome 21 silencing. Introducing an X-inactivation center on the extra chromosome in mouse models of DS will reveal whether correction of trisomy 21 is feasible in vivo.

The hypocellularity in the hippocampus and cerebellum in DS models has been corrected by treatment with an agonist of the sonic hedgehog (Shh) pathway that controls proliferation and differentiation during brain development [Das et al., 2013]. Feng Gao (USA) presented new mouse lines designed to produce inducible expression of Shh either in the hippocampus or in the cerebellum. Crossing these new lines with DS mouse models will allow testing the rescue of phenotypes after induction of Shh in either or both brain regions.

GABAergic over-inhibition has been shown to be linked to cognitive deficits in DS mouse models [Kle-
280

Mol Syndromol 2018;9:279-286 DOI: $10.1159 / 000494231$
Reeves et al. 
schevnikov et al., 2004]. GABA $\mathrm{A}$ receptors are permeant to chloride ions controlled by the chloride exchanger. The expression of the chloride importer NKCC1 is increased in DS [Deidda et al., 2015]. Andrea Contestabile (Italy) presented data showing that NKCC1 knock down in the hippocampus of DS mouse model, Ts65Dn, recues behavioral performance in learning and memory tests, opening new venues for the treatment of cognitive deficits with NKCC1 inhibitors.

GABAergic inhibition in DS can be corrected with antagonists of the $\mathrm{GABA}_{\mathrm{A}}$ receptors such as pentylenetetrazol [Fernandez et al., 2007] only when administered during daylight hours. Craig Heller (USA) showed results indicating that the suprachiasmatic nucleus activated during daylight, when lesioned in Ts65Dn mice, improved cognition, thus highlighting the role of the circadian system in these mice where memory consolidation is impaired by excessive GABAergic inhibition.

Alexander Kleschevnikov (USA) presented a study on the effects of chronic suppression of monoacylglycerol lipase (MAGL) with a selective inhibitor JZL184 on adult neurogenesis in the dentate gyrus of aged Ts65Dn mice. MAGL inhibition with JZL184 increased brain levels of 2-AG, restored long-term potentiation in hippocampal CA1, and improved long-term memory in Ts65Dn mice. Chronic treatment with JZL184 partially restored adult neurogenesis and improved cognition with no effect on thigmotactic behavior (an index of anxiety) in Ts65Dn mice. Thus, targeting of MAGL may be a promising therapeutic approach for cognitive improvement in DS.

Ana Velasco (Spain) presented her work on oleic acid as a neurotrophic factor that induces neuronal differentiation by increasing the expression levels of proteins such as GAP-43 and MAP-2. Analysis in a cellular model of DS (CTb cell line) indicated that oleic acid is preferably located in the cell membrane and promotes cell differentiation in euploid, but not trisomic cells. The amount of phosphatidylcholine and the expression of enzymes involved in phospholipid synthesis were also reduced in trisomic cells suggesting a marked deregulation of phosphatidylcholine biosynthetic pathways by trisomy.

Lotta Granholm (USA) presented her work on designer receptors and the locus coeruleus (LC) in Ts65Dn mice. Her studies showed that the LC degenerates in Ts$56 \mathrm{Dn}$ mice, a finding also observed in patients with DS. Loss of noradrenergic neurons in LC has been linked to increased amyloid aggregation, increased inflammation, and correlates with the severity of cognitive deficits. Stimulation of noradrenergic input using designer receptors exclusively activated by designer drugs (DREADD), ex-

The Second International Conference of the T21RS pressed using viral constructs, reduced hyperactivity and inflammation and improved novel object recognition in Ts65Dn mice. Thus, reducing hyperactivity in discrete neuronal populations may improve cognition and prevent neurodegeneration in DS.

In a study aimed to identify urinary biomarkers in individuals with DS with and without obstructive sleep apnea, Brian Skotko (USA) presented data indicating that the combination of the concentration of 4 neurotransmitters in the urine appear to have predictive value for obstructive sleep apnea with $90 \%$ sensitivity and $68 \%$ specificity [Elsharkawi et al., 2017].

\section{New Approaches to Stem Cell Research in Down Syndrome}

Human induced pluripotent stem cells (iPSCs) generated from somatic cells of individuals with specific diseases and disorders offer an important model system to reveal cellular and molecular events underlying pathogenesis as well as provide a means for initial assessments of potential therapeutic interventions. Thus, studies using iPSCs are especially valuable for enhancing our understanding of the cellular and molecular mechanisms of a complex condition such as DS.

Guo-li Ming (USA) discussed cerebral organoids and $3 \mathrm{D}$ cultures from iPSCs that provide a platform to investigate human brain development and disease conditions. She highlighted the effects of the Zika virus on the developing human brain and the link between Zika infection and microcephaly. Using miniature bioreactors to better mimic the growth environment and enhance the nutrient supply, Dr Ming showed that forebrain-specific organoids recapitulate key features of human cortical development and provide a platform to further understand molecular events and mechanisms underlying early brain development. These findings provide the groundwork and potential blueprint for elucidating neurodevelopmental mechanisms that go awry in DS.

Anita Bhattacharyya (USA) described the utility of patient-derived trisomy 21 iPSCs to model neurodevelopment in DS. Trisomy 21 iPSC-derived neural progenitors and neurons can recapitulate aspects of DS neuropathology. Modeling development of cortical neurons, both from dorsal and ventral forebrain, show that trisomy 21 progenitors and neurons have deficits that may lead to the paucity of neurons in the DS brain. Dr. Bhattacharyya also outlined the current challenges in the use of iPSC to model neural development including variability among

Mol Syndromol 2018;9:279-286 DOI: $10.1159 / 000494231$ 
DS individuals, heterogeneity of derived neurons, and immaturity of differentiated neurons. She introduced technologies that may address these challenges such as 3D organoids, CRISPR/Cas gene editing, signaling sensors, and designer receptors.

The application of cerebral organoids to study neural development in DS was presented by Tristan McClureBegley (USA). Analysis of 3D organoids derived from trisomy 21 iPSCs suggested reduced proliferation of neural progenitor cells. Deep proteome profiling showed alterations in Wnt and Notch signaling pathways, neurotransmitter metabolism, axon guidance, and cell adhesion. Pharmacological inhibition of the protein kinase, DYRK1A, changed the proteome profile with significant effects on expression levels of several key pathway members involved in neuronal differentiation and axon guidance.

Lin Tian (USA) focused on astrocyte function in DS. Astrocytes participate in brain development and support and modulate neuronal activity. Precise communication between neurons and astrocytes appears to play a critical role orchestrating higher brain functions. Using genetically encoded calcium sensors to monitor cellular function in trisomy 21 and control iPSC-derived astrocytes, she showed that DS astrocytes exhibit spontaneous calcium fluctuations and that neuronal excitability is decreased by DS astrocytes. The results suggest potential targets for therapeutics for DS and establish an optical neurophysiological platform for studying human astrocyte function in developing neural circuits.

Overall, these talks underscored the critical utility of human iPSCs to define the missteps in neurodevelopment and function in DS. Recent technological advances that are being used will stimulate the effort to better understand consequences of trisomy 21 on the human brain.

\section{Clinical Trials Targeting Cognition in Down Syndrome}

Xavier Liogier d'Ardhuy (Switzerland) described the results of the Roche clinical trial based on observations of excessive GABA $_{\mathrm{A}}$ inhibitory signal in models of DS. This phase 2 trial investigated the efficacy of basmisanil, a $\mathrm{GABA}_{\mathrm{A}} \alpha 5$ negative allosteric modulator. This multicenter trial included 156 young adults with DS randomized to receive either placebo, $120 \mathrm{mg}$, or $240 \mathrm{mg}$ basmisanil for 26 weeks. As shown by various safety parameters including EEG recordings, this compound was safe and well tolerated. Target engagement was demonstrated.
During this trial, suitable cognitive and functioning scales as well as composite scores were evaluated. There was no statistically significant change from baseline or between groups in favor of basmisanil for cognitive domain scores. Thus, the primary and secondary endpoints on improvement in cognition and functioning were not met.

Rafael de la Torre (Spain) presented a phase 2 clinical trial based on preclinical studies showing effects of green tea extract containing EGCG, an inhibitor of DYRK1A activity, and cognitive stimulation on cognitive ability. This trial (TESDAD study) compared 2 groups: one receiving EGCG (tea extract with 45\% EGCG) and cognitive training, the second receiving placebo and cognitive training for 12 months with a 6 month follow-up after treatment discontinuation. The primary outcome was the change from baseline at 12 months in cognitive and functional components of the TESDAD neuropsychology battery, which included measures of attention, psychomotor speed, memory, executive functions, language, adaptive behavior, quality of life, quality of sleep, and neuropsychiatric symptoms. EGCG plus cognitive training was significantly more effective than placebo at improving scores for visual recognition memory, inhibitory control, adaptive behavior, and a Global Clinical Improvement Index.

Cecile Cieuta-Walti (France) described rational and objectives of the PERSEUS study which will involve 4 centers in Spain and 1 center in France. This study will be performed with children (6-12 years) at an age where cerebral plasticity is maximal. Safety of pure EGCG has been evaluated in preclinical studies assessing effects of 3 different doses on hepatic and cardiac functions of mice. Safety and tolerability of EGCG will be evaluated for 6 months with a panel of biochemical markers. Secondary objectives will allow to measure effects of this treatment on cognitive performance and adaptive functionality of children with DS.

Chrysanthy Ikonomidou (USA) reported on trials designed to assess safety and efficacy of transcranial direct current stimulation (tDCS). High definition tDCS enables noninvasive electrical stimulation of the brain. It has been shown to improve some cognitive functions in humans. Results of a phase 1 safety trial on healthy adults after 4 networks were stimulated indicated no abnormalities on physical and neurological examination, or on electrocardiogram and electroencephalogram. Preliminary EEG results suggest that tDCS may induce long-lasting changes in excitability in the human brain. This study will be followed by a phase 2 trial to examine these parameters and cognitive parameters (ImPACT) in 5- to 10-yearold children with DS.
Reeves et al. 
Sarah Hart (USA) presented a review of the progression from preclinical studies with mouse models to human clinical trials research using pharmacological interventions to improve cognition and adaptive functioning in DS. Challenges identified included interindividual variability with potential for subset of responders, age of participants of previous studies (mainly young adults), limited evidence of efficacy, and lack of standard endpoints to assess efficacy, particularly in developing populations with risk of ceiling/floor effects.

\section{Alzheimer Disease in Down Syndrome - Neuropathology and Disease Mechanisms}

Ira Lott (USA) gave an overview of $\mathrm{AD}$ pathogenesis and potential biomarkers in people with DS. He described "J.J.S.," a high-functioning elderly person with partial trisomy 21 who had a deletion of the APP region in the third copy of chromosome 21. J.J.S. had no amyloid plaque deposition and did not progress to dementia, providing support that triplication of APP is necessary for AD dementia in people with DS. Changes in white matter integrity, apoptosis-related proteins, oxidative stress, neuroinflammation, diabetes, and seizures were cited as possible contributors to AD in DS. Baseline amyloid PET scans were suggested to be useful for predicting the transition to dementia. Dr. Lott discussed evidence showing that $\mathrm{A} \beta 42$ is reduced in cerebrospinal fluid (CSF) and increased in neuronally derived exosomes. Finally, results presented suggested that $A \beta$ is retained in the brains of non-demented people with DS but released when those same individuals convert to dementia.

Francis Wiseman (UK) reported the phenotype of offspring from a mouse model of DS containing a partial Hsa21 (the Tc1 mouse, which does not contain a third functional copy of APP) crossbred with J20 hAPP transgenic mice. She found that the presence of the extra $\sim 200$ genes in the $\mathrm{Tc} 1$ mice increased $\mathrm{A} \beta$ plaque deposition in the cortex and hippocampus. The largest increase was in $A \beta 42$ levels, which correlated with reductions in $A \beta 38$ and $A \beta 40$. APP processing was shifted similar to that seen in PS1 mutants; however, no differences were observed in gamma-secretase activity. Reduced cathepsin B, a lysosomal enzyme, but not cathepsin $\mathrm{D}$, was observed in DS fibroblasts and thought to contribute to the increased $A \beta$ load. Thus, trisomy of genes other than APP appear to influence $\mathrm{AD}$ pathology in people with DS.

Eugenio Barone (Italy) discussed insulin resistance in $\mathrm{AD}$ and suggested that it may be independent of $\mathrm{A} \beta \mathrm{pa}-$

The Second International Conference of the T21RS thology in AD. Ts65Dn mice at 1, 3, 9, and 18 months of age exhibited increased pAkt/Akt at 1 and 9 months and elevated GSK3 $\beta$ at 1,3 , and 9 months. Inhibition of the insulin response element reduced PTEN and SIRT1 causing a rise in insulin resistance. Thus, impairment of insulin signaling appears to be an early event in the Ts65Dn mouse model.

Fiorenza Stagni (Italy) reported beneficial effects of 7,8-dihydroxyflavon (DHF), a naturally-occurring flavonoid that crosses the blood brain barrier and binds the TrkB receptor, in both in vitro and in vivo studies. DHF increased the acquisition of neuronal phenotypes $\left(\mathrm{MAP}^{+}\right)$in trisomic neuronal progenitor cells and promoted neurite elongation, but did not affect proliferation. In in vivo studies, DHF increased proliferation of dentate gyrus neurons between $\mathrm{P} 3$ and $\mathrm{P} 15$, and increased pTrkB, pERK1, and pERK2 levels at P15. DHF restored neuron number and synaptic density in the dentate gyrus of adult mice and improved learning and memory in the Morris water maze at $\mathrm{P} 45$.

Meifang Xiao (USA) reported that neuronal pentraxin 2 (NPTX2), which is regulated by synaptic activity and mediates activation of parvalbumin interneurons, is reduced at both the mRNA and protein levels in mid-frontal gyrus in brains of 19- to 40-year-old people with DS and DSAD. CSF NPTX2 levels are reduced in AD and correlate with cognitive status in $\mathrm{AD}$, but not in people with AD pathology without dementia. She suggested that changes in NPTX2 may be associated with the mechanism for the transition from AD pathology to dementia in people with $\mathrm{DS}$ and in people with $\mathrm{AD}$ pathology without dementia.

\section{Alzheimer Disease in Down Syndrome - Cognition, Biomarkers, and Clinical Research}

Wayne Silverman (USA) discussed the benefit of including full-scale IQ scores throughout life to help assess conversion to dementia in individuals with DS. IQ scores can be used to track cognitive status over long periods of time and help to discern changes in cognition within individuals.

Alain Dekker (The Netherlands) discussed the behavioral and psychological symptoms associated with dementia in DS. He assessed frequency and severity of behavioral changes in a large cross-sectional study in people with DS without dementia $(n=149)$, people with DS with questionable dementia $(n=65)$, and people with DS with Alzheimer dementia $(n=67)$. The proportion of individ-

Mol Syndromol 2018;9:279-286

DOI: $10.1159 / 000494231$ 
uals presenting an increased frequency of symptoms of anxiety, sleep disturbances, agitation and stereotypical behavior, aggression, apathy, depressive symptoms, and eating/drinking alterations was highest in the group with dementia and lowest in the group without dementia.

Brad Christian (USA) presented findings of a collaborative project between the Universities of Pittsburgh and of Wisconsin-Madison to measure and document amyloid deposition in adults with DS without symptoms of dementia (age $\geq 30$ years). DS individuals were followed at 3-year intervals to understand the course of amyloid deposition and its effect on functioning over time.

Ai-Ling Lin (USA) presented the results of a study using proton magnetic resonance spectroscopy to measure neuronal and glial metabolites in the posterior cingulate cortex of 22 adults with DS and in 15 age- and gendermatched healthy controls. The data indicates the presence of an overall higher glial inflammatory component in DS prior to and possibly independent of the presence of dementia.

Finally, Michael Raffi (USA) presented Tau PET data on 9 adults with DS and compared those findings with FDG PET, T1 MRI, and amyloid PET results from those same individuals. Tau accumulation was found to be associated with amyloid positivity and age as well as with progressive neurodegeneration as measured using FDG (hypometabolism) and MRI (atrophy).

European Collaborative Studies of Alzheimer Disease in Down Syndrome

Andre Strydom (UK) described the Horizon 21 study in which DNA is being collected from 300 people with DSAD and approximately 700 people with DS (without $\mathrm{AD})$. Biomarkers and brain imaging are being analyzed, and the study also involves industry collaborators. The objectives are to harmonize clinical data, better understand $\mathrm{AD}$ in people with DS, identify non-DS contributions, and look for association with GWAS-relevant AD risk genes. Dr. Strydom also discussed DS1000, a clinical study of prevention of $\mathrm{AD}$ in people with $\mathrm{DS}$ and cited the need for harmonization of protocols, reliable outcome measures, drug tolerability, disease progression, and the adoption of an adaptive trial platform.

Tonnie Coppus (The Netherlands) addressed the challenges in harmonizing protocols across different trial centers, including dementia rating. The goal of these large clinical studies is to identify mechanisms and predictors of dementia in people with DS. European sites include Paris, London, Cambridge, Barcelona, and the Netherlands. She discussed the need for standardization of phys- ical and mental status exams, cognitive measurements, etc. Demographics as well as longitudinal assessments of dementia and biomarkers (blood, CSF, MRI, and PET) from baseline to 1- and 2-year follow-up visits are integral to this study. Challenges include language issues (i.e., translation across many languages beyond English and Spanish) and selection, sensitivity, and specificity of cognitive tests.

Shahid Zaman (UK) described the "DiDS" (Defeat Dementia in DS) initiative, which involves brain imaging, EEG, retinal markers, and mitochondrial function tests. $\mathrm{He}$ also discussed AD-related temporal changes in DS brain and suggested that while the general pattern (also known as Jack model) is similar to AD, the changes occur much earlier. Interestingly, thicker cortices were observed in PiB-negative people with DS than non-DS controls; however, in the presence of amyloid (PiB-positive), cortical thinning and a posterior progression of $\mathrm{AD}$ pathology was observed in DS brains.

Juan Fortea (Spain) described the DABNI (Down Alzheimer Barcelona Neuroimaging Initiative) study, which is similar to ADNI (Alzheimer Disease Neuroimaging Initiative). $\mathrm{He}$, too, described $\mathrm{AD}$ as a "posterior story" in people with DS, citing early posterior changes in hypometabolism by FDG-PET imaging. Dementia in people with DSAD was associated with reduced FDGPET and increased amyloid PET and vascular amyloid (CAA), although CSF A $\beta 40$ levels did not distinguish subjects with or without CAA. He promoted the idea of a "virtuous" cycle in which bidirectional flow of information between sporadic AD and DSAD would benefit both populations.

\section{T21RS Science \& Society Symposium}

Based on a strong belief that scientific research will aid the understanding of DS and benefit the DS community, the T21RS Committee for Science \& Society works together with local and (inter)national DS associations in a mutual way. In addition to regularly publishing T21RS Science \& Society Bulletins, i.e., updates in understandable language about the current scientific status of a particular aspect of DS, the committee organizes the bi-annual T21RS Science \& Society Symposium. After a very successful kick-off symposium in Paris in 2015 [Delabar et al., 2016], the second edition was organized during the 2nd T21RS International Conference in Chicago.

Presented by Peter De Deyn and Alain Dekker (The Netherlands), the symposium's theme was "Down syn-
Reeves et al. 
drome associations as research partners," and it was aimed at sharing thoughts and ideas, and promotes discussion about clinical scientific research and future directions. Diana Bianchi (USA), director of the National Institute of Child Health and Human Development (NICHD, NIH), chaired a debate about "The pros and cons of having my relative with DS participate in clinical research." Parents of children with DS shared their thoughts and experiences with their child participating in clinical studies. Whereas all parents were truly in favor of scientific research, they were not all positive about participating in clinical trials. The CLEMATIS clinical trial by Roche using the $\mathrm{GABA}_{\mathrm{A}} \alpha 5$ negative allosteric modulator, basmisanil, had recently been terminated, and several parents elaborated on their experiences with the trial. A number of parents reported positive effects in their child and, therefore, did not understand why the trial was stopped. Whereas Roche concluded a lack of efficacy on selected outcome measures, these parents had wanted to continue (although they were blinded to whether receiving the drug or placebo). The debate clearly illustrated the gap between science on the one hand and society on the other hand, pointing at 2 different perspectives (group level vs. individual level) and the need that such decisions (e.g., end of a trial) are explained in clear terms to all participants. In addition, it was highlighted that more accessible information with regard to scientific methodological aspects of clinical research should be disseminated.

Following the debate, Juan Fortea (Spain) and Cynthia Lemere (USA) cochaired a session on "Medical policies for people with DS," including initiatives to integrate care and research with social aspects for people with DS and their families. Sebastian Videla (Spain) presented the health plan initiative for medical comorbidities related to aging in DS. This collaboration of the Hospital of Santa Creu i Sant Pau and the Catalonian Down Syndrome Foundation offers free screening and, if necessary, specialized care but also allows people to participate in clinical research associated with this health plan. Next, Melissa Parisi (NIH, USA), presented an update on the NIH efforts to facilitate research on DS. One of the key initiatives concerns DS Connect, a registry in the US enabling people with DS and their families to connect to researchers and health care provides, participate in surveys, and express interest for clinical studies. Michelle Whitten (USA, Global Down Syndrome Foundation) elaborated on "Medical care for adults with DS-lifting barriers," focusing on existing barriers that people with DS may face on a daily basis. Whitten provided examples of the 7 most

The Second International Conference of the T21RS common barriers (attitudinal, communication, physical, policy, programmatic, social, and transportation barriers) and stressed the importance of developing medical care guidelines for adults with DS. Finally, Kandi Pickard (USA, National Down Syndrome Society) presented a progress report about the "Achieving a Better Life Experience (ABLE) Act," which was signed into law in 2014 in the USA. This law allows disabled individuals to open a tax-exempt savings account to cover for a variety of essential expenses associated with maintaining health, independence, and quality of life, including costs related to education, health care, and job training.

The second half of the symposium was devoted to presentations by attending DS associations from different countries. A total of 15 organizations introduced themselves and described their contribution to research and core initiatives. The large variety of clinical, educational, and social research initiatives that are currently undertaken was impressive and strongly illustrated the passion and efforts by the DS community. The presentations of the associations can be downloaded via http:// t21rs.org/science-society/t21rs-science-society-symposium-2017.

The symposium highlighted exemplary results when combining the strengths of both science and society but also touched on sore spots that need further improvement in the future.

The International Conference of the T21RS has become an established forum that brings together researchers, clinicians, organizations, and families with the prime objective of accelerating research, improving the quality of life, and ultimately providing therapeutic answers to the most pressing needs of persons with DS.

\section{Acknowledgments}

The 2nd International Conference of the Trisomy 21 Research Society was made possible by the generous support of Global Down Syndrome Foundation (presenting sponsors), the Jerome Lejeune Foundation, Lumind RDS-Foundation, the Matthews Foundation, Trisomie 21 France (main sponsors), the National Institutes of Health, NINDS, NCHD and NIA (R13 NS100380 to J.B.), the National Down Syndrome Society, Down Syndrome International, Alzheimer Association and Down Syndrome Association UK and robiodrug AG. T21RS is the first international nonprofit research society on Down syndrome, founded on the 17th of April 2014 in Groningen, the Netherlands. During the T21RS International Conference, the society also held its annual General Assembly meeting. Annual reports, conference material, and other information about the society can be found at http://www.t $21 \mathrm{rs}$. org. 


\section{References}

Das I, Park J-M, Shin JH, Jeon SK, Lorenzi H, et al: Hedgehog agonist therapy corrects structural and cognitive deficits in a Down syndrome mouse model. Sci Transl Med 5:44-53 (2013).

Deidda G, Parrini M, Naskar S, Bozarth IF, Contestabile A, Cancedda L: Reversing excitatory $\mathrm{GABA}_{\mathrm{A}} \mathrm{R}$ signaling restores synaptic plasticity and memory in a mouse model of Down syndrome. Nat Med 21:318-326 (2015).
Delabar JM, Allinquant B, Bianchi D, Blumenthal $\mathrm{T}$, Dekker A, et al: Changing paradigms in Down syndrome: the first International Conference of the Trisomy 21 Research Society. Mol Syndromol 7:251-261 (2016).

-Elsharkawi I, Gozal D, Macklin EA, Voelz L, Weintraub G, Skotko BG: Urinary biomarkers and obstructive sleep apnea in patients with Down syndrome. Sleep Med 34:84-89 (2017).
Fernandez F, Morishita W, Zuniga E, Nguyen J, Blank M, et al: Pharmacotherapy for cognitive impairment in a mouse model of Down syndrome. Nat Neurosci 10:411-413 (2007).

- Jiang J, Jing Y, Cost GJ, Chiang JC, Kolpa HJ, et al: Translating dosage compensation to trisomy 21. Nature 500:296-300 (2013).

Kleschevnikov AM, Belichenko PV, Villar AJ, Epstein CJ, Malenka RC, Mobley WC: Hippocampal long-term potentiation suppressed by increased inhibition in the Ts65Dn mouse, a genetic model of Down syndrome. J Neurosci 24:8153-8160 (2004). 